D KIDNEY FIBROSIS

\section{Cyclin G1 drives maladaptive renal repair}

Following acute kidney injury (AKI), damaged renal epithelial cells secrete profibrogenic factors that contribute to the damage repair response but can also lead to chronic kidney disease (CKD). Now, Joseph Bonventre and colleagues describe how cyclin G1 (CG1), which leads to cell cycle arrest in response to DNA damage, also promotes renal fibrosis.

"Our previous work showed that kidney injury leads to a maladaptive repair process, which results in cell cycle arrest at the G2-M phase of the cell cycle and induces profibrogenic cytokines," explains Bonventre. To understand the link between cell cycle arrest and fibrosis, the researchers analysed differences in gene expression between rats injected with aristolochic acid, which induces severe kidney fibrosis, and control rats injected with vehicle; the $\mathrm{p} 53$ pathway was one of the most upregulated pathways and researchers had previously linked it to fibrosis and cell cycle arrest in mouse models of injuryrelated fibrosis. Ccng1, which encodes CG1 and is transcriptionally regulated by $\mathrm{p} 53$, was highly upregulated in those mouse models of fibrosis;

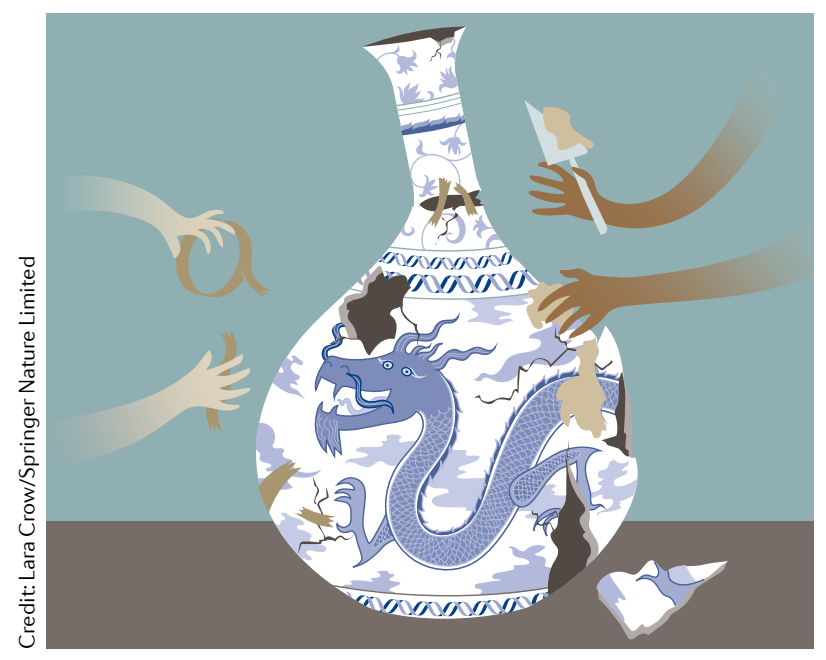

this atypical cyclin regulates cell cycle arrest at G2-M. The researchers also observed that in multiple mouse models of injury-related fibrosis, many proximal tubular cells in damaged kidneys contained target of rapamycin (TOR)-autophagy spatial coupling compartments (TASCCs). These cellular compartments are characteristic of senescent cells and result from the fusion of late autophagosomes with lysosomes that contain mTORC1; amino acids released from the autophagosome activate mTORC1 and promote the senescence-associated secretion programme. Analysis of human renal biopsy samples showed that renal epithelial cells in the G2-M phase from patients with AKI or CKD were enriched for TASCCs, and the presence of these structures correlated with tissue fibrosis.

"We complemented mouse in vivo studies and the analysis of human tissue with cell culture techniques in which HK2 and LLC-PK1 cell lines were used to explore various mechanistic pathways," clarifies Bonventre. Treatment of HK2 cells with aristolochic acid not only induced CG1 expression, but most of the cells that stained with phospho-histone $\mathrm{H} 3$ ( $\mathrm{pH} 3$ ), a marker of the G2-M phase of the cell cycle, also contained TASCCs; treatment also induced the secretion of connective tissue growth factor. Similarly, transfection of HK2 cells with CG1 led to an increase in $\mathrm{pH}^{+}$cells, which were also TASCC ${ }^{+}$, and promoted the secretion of profibrinogenic connective tissue growth factor and transforming growth factor- $\beta$ (TGF $\beta$ ). Conversely, deletion of Ccng1 in mice led to reduced formation of TASCCs and an attenuated fibrotic response to aristolochic acid compared with wild-type mice. Moreover, transfection of LLC-PK1 cells with a dominant negative form of Rag GTPase, which prevents the recruitment of mTORC1 to the lysosome and thereby prevents TASCC formation, reduced the secretion of connective tissue growth factor in response to aristolochic acid.

Of note, the researchers observed the same pattern of increased cell cycle arrest at G2-M, TASCC formation and increased CG1 expression in a model of liver fibrosis. "These results suggest a common pathway that promotes organ fibrosis in response to DNA damage," remarks Bonventre.

To gain further insight into the role of TASCCs in renal fibrosis, the researchers used mice with inducible, proximal tubule-specific deletion of the mTORC1 component, Raptor. Proximal tubule-specific deletion of Raptor 21 days after the induction of ischaemia-reperfusion or aristolochic acid-induced injury resulted in reduced expression of fibrosis markers compared with control mice. Despite the reduction in fibrosis, the absence of Raptor did not prevent the induction of cell cycle arrest and upregulation of CG1 in response to renal injury; the main difference was that Raptor deletion reduced the number of cells at G2-M that contained TASCCs.

Cell cycle arrest at G2-M might also be linked to dedifferentiation of proximal tubular cells, as treatment of LLC-PK1 cells with aristolochic acid or their transfection with CG1 led to loss of proximal tubular cell markers and increased vimentin expression.

"We will now attempt to target CG1 or enhance the apoptosis of DNA-damaged cells," adds Bonventre. "These approaches should reduce the number of cells in G2-M arrest and attenuate fibrosis."

Monica Wang

ORIGINAL ARTICLE Canaud, G. et al. Cyclin G1 and TASCC regulate kidney epithelial cell G2-M arrest and fibrotic maladaptive repair. Sci. Transl Med. 11, eaav4754 (2019) 